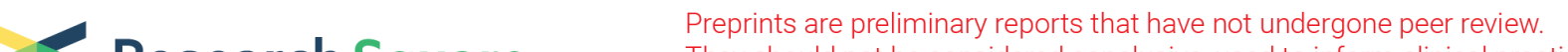 $\begin{array}{ll}\text { Research Square } & \begin{array}{l}\text { They should not be considered conclusive, used to inform clinical practice, } \\ \text { or referenced by the media as validated information. }\end{array}\end{array}$
}

\section{Modification of the Luedeking and Piret Model with a Delay Time Parameter for Biotechnological Lactic Acid Production}

María Carla Groff ( $\square$ mcarlagroff@gmail.com )

Universidad Catolica de Cuyo https://orcid.org/0000-0002-7133-1582

\section{Gustavo Scaglia}

Universidad Nacional de San Juan Facultad de Ingenieria

Oscar A. Ortiz

Universidad Nacional de San Juan Facultad de Ingenieria

Sandra E. Noriega

Universidad Catolica de Cuyo

\section{Research Article}

Keywords: Luedeking and Piret, First Order Plus Dead Time Model, lactic acid, fermentation

Posted Date: October 19th, 2021

DOI: https://doi.org/10.21203/rs.3.rs-892915/v1

License: (c) (i) This work is licensed under a Creative Commons Attribution 4.0 International License. Read Full License

Version of Record: A version of this preprint was published at Biotechnology Letters on January 29th, 2022. See the published version at https://doi.org/10.1007/s10529-022-03227-0. 
1 Modification of the Luedeking and Piret Model with a delay time parameter for

2 biotechnological lactic acid production.

3 M. Carla Groff ${ }^{1,2^{*}}$, Gustavo Scaglia ${ }^{2,3}$, Oscar A. Ortiz ${ }^{3}$, Sandra E. Noriega ${ }^{1}$.

$4{ }^{1}$ Instituto de Investigaciones en Ciencias Químicas. Facultad de Ciencias Químicas y Tecnológicas,

5 Universidad Católica de Cuyo, San Juan, Argentina.

$6 \quad{ }^{2}$ Consejo Nacional de Investigaciones Científicas y Técnicas (CONICET).

$7 \quad{ }^{3}$ Instituto de Ingeniería Química, Universidad Nacional de San Juan, San Juan, Argentina.

$8 *$ Corresponding author:

$9 \quad$ Tel.: +5492645 436303

10 E-mail: mcarlagroff@gmail.com

11 Address: Instituto de Investigaciones en Ciencias Químicas, Facultad de Ciencias Químicas y Tecnológicas, 12 Universidad Católica de Cuyo. Av. Ignacio de la Roza 1516 Oeste, Rivadavia. CP 5400. San Juan. 13 ARGENTINA.

Acknowledgments The authors thank the Consejo Nacional de Investigaciones Científicas y Técnicas de Argentina (CONICET), the Universidad Católica de Cuyo and the Secretaría de Ciencia, 16 Tecnología e Innovación (SeCiTI, San Juan) for the financial support provided to the doctoral fellow M. Carla Groff 


\section{Abstract}

Objectives To obtain a mathematical model that adequately describes the time lag between biomass generation and lactic acid production of lactic fermentations.

Methods Seven experimental kinetics from other research works were studied to validate our proposal: four studies of Fungal Submerged Fermentation and three cases of Bacterial Submerged Fermentation, including the data recollected by Luedeking and Piret.

Results We introduce a modification to the Luedeking and Piret model that consist in the introduction of a time delay parameter in the model, this parameter would account for the lag time that exists between the production of biomass and lactic acid. It is possible to determine this time delay in a simple way by approximating the biomass and product formation considering that they behave as a first order plus dead time system. The duration of this phenomenon, which is not described with the classical Luedeking and Piret model, is a function of microorganism physiology (ease of biomass growth), environment (nutrients) and type of inoculum.

Conclusion The Luedeking and Piret with delay model applications reveal an increase of the $\mathrm{R}^{2}$ in all cases, evidencing the quality of fit and the simplicity of the method proposed. These model would improve the accuracy of bioprocess scaling up.

Keywords: Luedeking and Piret; First Order Plus Dead Time Model; lactic acid; fermentation. 
The concept of bioeconomy has emerged several years ago as an eco-friendly alternative to stop using petrochemicals as precursors in chemical synthesis, and having the goal to use and revalue biomass, including lignocellulosic waste from agroindustry, as a solid substrate for obtaining a wide range of biosubstances. The objectives of bioeconomy are: sustainable development and circular economy (Bugge et al. 2016). One type of bioproduct that has been intensively studied in recent years is the lactic acid (LA). Lactic acid is a natural occurring organic acid, it has applications in pharmaceutical, cosmetic, chemical and food industry (Gündüz 2005). Lactic acid has also received attention for its use as a monomer in the production of polylactic acid, a completely biodegradable polymer (Herryman Munilla and Blanco Carracedo 2005; Inkinen et al. 2011). Lactic Acid had a world demand in 2016 of 1,220 kilo-ton (Grand View Research 2019). Bacterial and fungal strains are utilized to produce LA. Depending on the culture medium used, they can be classified into submerged liquid fermentations (homogeneous medium) and solid substrate fermentations (heterogeneous medium), basically differentiated by the liquid water content of 100 $\%$ and 60-70\%, respectively (Webb and Manan 2017).

For an industrial-scale Bioprocess to become a reality, an appropriate scale-up of the system must be carried out. Mathematical modeling is a very useful tool to achieve an adequate bioprocess scaling up and predicting bioreactor performance (Crater and Lievense 2018; Gonzalez et al. 2016). In order to develop a mathematical model for the process under consideration is necessary to take into account submodels that describe the kinetics, mass and energy transfers. The submodels that describes the kinetics equations involved in the biomass growth and LA production are the first and more important to be developed (Mitchel et al. 2006). The Logistic Model (L model) is the most widely used to describe the microbial growth, as it shows a good fitting in most of the microbial growth cases (Germec and Turhan 2020; Mohsin et al. 2019; Saat et al. 2014). And for the production of LA, the mathematical model with highest application rate is the Luedeking and Piret Model (LP model), which relates metabolite production to biomass concentration and microbial growth rate and is independent of substrate concentration (Luedeking and Piret 1959; Mitchel et al. 2006). It is also possible to apply First Order Plus Dead Time model (FOPDT model), which is widely used in the field of process control due to its simplicity and reproducibility (Arino et al. 2006; Sardella et al. 2020), but which have not been studied thus far in lactic fermentations either homogeneous or heterogeneous. 
Generally, the metabolite generation starts after a certain amount of biomass has been produced, observing a time lag between both kinetic curves. This lag time depends on the microbial metabolism that is being developed under certain experimental conditions. On one hand, the knowledge of this delay time would allow a simpler study of microbial metabolism, on another hand it would permit to perform a time optimization to obtain the desire metabolite by varying different factors (nutrients, temperature, $\mathrm{pH}$, among others). This phenomenon is omitted in the LP model, as there is no parameter that takes into account this delay time. Other researchers have proposed modifications to the LP model. (Amrane 2001) added an empirical term that considers the residual content of the limiting carbon substrate (lactose) in LA production using Lactobacillus helveticus. (Bouguettoucha et al. 2007) instead of using the residual lactose content, used a term with the limiting lactose concentration using the same microorganism to generate LA. Balannec et al. (2007) incorporated a term that takes into account the inhibitory effect of the undissociated form of LA and the inhibitory concentration of LA in fermentations with Lactobacillus helveticus. Rosero-chasoy et al. (2020) observed that the fermentation of complex substrates (agro-industrial waste) generates a delay time in the generation of Lactobacillus casei biomass due to an acclimatization time, so they incorporated a time delay term in the fermentation kinetics into the model. This lag time is not precisely the same as the one we propose in the present work, since the lag time we observe is between the time when the biomass starts to be generated and the time when the LA starts to be produced.

In this article, we analyzed the kinetic information available in literature of microbial growth and LA production from other research works. The experimental data extracted from those articles were mathematically modeled. In all cases, a delay time was observed between the microbial kinetic curves and the LA production, which motivated our proposal to modify the LP equation, improving the fitting of the LA production curves in all cases.

\section{Material and Methods}

\section{Compilation of experimental information from other research work}

Other research was used as a basis for presenting graphs of the kinetics of biomass growth and LA production of different fermentation systems using different carbonaceous substrates, whether amylaceous and lignocellulosic residues or simply glucose. We have differentiated between works using LAB as inoculum and those using fungi of the genus $R$. oryzae. The methodologies applied in each work are described in the following paragraphs. 

thermophilic and homofermentative $\mathrm{LAB}$, carrying out the fermentation at $45^{\circ} \mathrm{C}$ and keeping the $\mathrm{pH}$ constant at 6.0 (with sodium carbonate). The liquid culture medium was an aqueous solution with $5 \%$ anhydrous dextrose, $3 \%$ dehydrated yeast extract and mineral salts. They used a two liter closed fermenter with temperature, $\mathrm{pH}$, and $\mathrm{CO}_{2}$ controllers. To exclude atmospheric $\mathrm{O}_{2}$, the surface was covered with $\mathrm{CO}_{2}$.

Jin et al. (2005) used different agroindustrial wastes, including corn, pineapple, potato and wheat waste streams, each containing approximately $20 \mathrm{~g} / \mathrm{l}$ of starch or sugars, supplemented with peptone, yeast extract, $\mathrm{KH}_{2} \mathrm{PO}_{4}$, and $\mathrm{MgSO}_{4} \cdot 7 \mathrm{H}_{2} \mathrm{O}$. Fermentations were carried out at $30^{\circ} \mathrm{C}$ in $250 \mathrm{ml}$ Erlenmeyer flasks with shaking (150 rpm), using $100 \mathrm{ml}$ of culture medium, inoculated with $5 \mathrm{ml}$ of a spore suspension of $R$. oryzae $2062\left(10^{5}\right.$ spores/ml).

Palaniraj and Nagarajan (2012) used Lactobacillus casei MTCC 1423 (L. casei) as inoculum (6 x $10^{9} \mathrm{cfu} / 100 \mathrm{ml}$ ), which is a homofermentative and anaerobic acid tolerant LAB. The medium was a water solution with $100 \mathrm{~g} / \mathrm{l}$ potato waste, $12 \mathrm{~g} / \mathrm{l}$ of yeast extract, $3 \mathrm{~g} / \mathrm{l}$ of ammonium chloride (for burring) and 10 $\mathrm{ml} / \mathrm{l}$ of enzymes ( $\alpha$-amylase and glucoamylase). The fermentation was carried out in flasks at $37^{\circ} \mathrm{C}, \mathrm{pH} 6.5$ and for $60 \mathrm{~h}$.

Berry et al. (1999) used Lactobacillus rhamnosus ATCC 10863 (homofermentative) to produce LA. The fermentation was carried out in fermenters of 2 liter working volume (Applikon BV, Netherlands). Five milliliters of inoculum (concentration was not mentioned in the paper) was added in $200 \mathrm{ml}$ of culture medium. The culture medium contained glucose enriched with a wide variety of minerals salts, vitamins and aminoacids. The fermentation was carried out at $40{ }^{\circ} \mathrm{C}$, with agitation at $110 \mathrm{rpm}$, without aeration and maintaining the $\mathrm{pH}$ constant at 5.5.

\section{Mathematical modeling}

\section{Microbial growth}

In this work, we propose the use of an alternative mathematical model to fit de kinetic growth of different fermentative process. Microorganisms have an adaptation time in their first metabolic phase of development, showing a lag phase, where the cells are adapting to their environment and may also involve the synthesis of adaptive enzymes. The duration of the lag time is very important to be determined in fermentation processes. The length of this phase depends on the physiology of the microorganism, the environmental condition, the type and amount of inoculum, even the conditions under which the 
124

125

126

127

propagation of the micro-organism was developed before fermentation and also on the kind of fermentation that is being performed (Rodríguez León et al. 2017). Another mathematical model that can responds to this phenomenon is the FOPDT model also largely used in process control (Arino et al. 2006; Sardella et al. 2020) :

$$
\frac{d X}{d t}+\frac{X}{T_{p}}=\frac{1}{T_{p}} \cdot X_{\max }\left(t-T_{0}\right), \quad X(0)=X_{0}
$$

Where the function $X_{\max }$ is defined by:

$$
X_{\text {max }}\left(t-T_{0}\right)=\left\{\begin{array}{lll}
X_{\max } & \text { for } & t \geq T_{0} \\
0 & \text { for } & t<T_{0}
\end{array}\right.
$$

The solution to the equation (1) it is shown in equation (3):

$$
X(t)=\left\{\begin{array}{lr}
C \cdot \exp ^{-\left(t-T_{0}\right) / T_{p}}+X_{\max } & \text { for } t \geq T_{0} \\
X=X_{0} & \text { for } t<T_{0}
\end{array}\right.
$$

In order to find the integration constant $\mathrm{C}$, for $\mathrm{t}=T_{0}$ :

$$
X(0)=X_{0}=C+X_{\max } \rightarrow C=X_{0}-X_{\max }
$$

Replacing (4) in (3):

$$
X(t)= \begin{cases}\left(X_{0}-X_{\max }\right) \cdot \exp & \text { for } t \geq T_{0} \\ X=X_{0}\left(t-T_{0}\right) / T_{p}+X_{\max } & \text { for } t<T_{0}\end{cases}
$$

Rearranging:

$$
X(t)= \begin{cases}X_{0} \cdot \exp ^{-\left(t-T_{0}\right)} / T_{p}+X_{\max }\left(1-\exp ^{-\left(t-T_{0}\right) / T_{p}}\right) & \text { for } t \geq T_{0} \\ X=X_{0} & \text { for } t<T_{0}\end{cases}
$$

Where dX/dt: Biomass growth rate $\left[\mathrm{g} \cdot \mathrm{l}^{-1} \cdot \mathrm{h}^{-1}\right] ; \mathrm{X}(\mathrm{t})$ : Fungal Biomass concentration obtained for a specific amount of time $\left[\mathrm{g} . \mathrm{l}^{-1}\right] ; X_{\max }$ : Maximum biomass concentration $\left[\mathrm{g} . \mathrm{l}^{-1}\right] ; X_{0}$ : Initial biomass concentration or inoculum $\left[\mathrm{g} .1^{-1}\right], \mathrm{t}$ : Time $[\mathrm{h}] ; T_{0}[\mathrm{~h}]$ is the parameter which provides a quick and easy way to find out the approximate duration of the latency phase, and $T_{p}[\mathrm{~h}]$ is a parameter of the process which provides information on the speed of growth up to $X_{\max }$. In this FOPDT model, the microbial growth profile 
does not show an acceleration stage between the lag phase and the exponential phase, as described by the

145

146

147

148

149

150

151

152

154

155

$\mathrm{L}$ model, so the $T_{0}$ parameter would include both stages, the adaptation phase and the cell acceleration phase.

In the FOPDT model, when $T_{0}=0$, the model takes the form of the First Order model (FO) but as microbial growth kinetics rarely occur without a lag phase, this is why $T_{0}>0$ generally, and therefore the system can be modelled with the FOPDT model. In the case of microbial kinetic the stationary state is reached when $X_{\max }=$ constant.

In the FOPDT model the parameters $T_{0}$ and $T_{p}$ are obtained with the equations 7 and 8 (Sardella et al. 2020):

$$
T_{p}=1.5\left(t_{2}-t_{1}\right)
$$

$$
T_{0}=t_{2}-T_{p}
$$

Where $\mathrm{t}_{1}$ is the time [h] in which $28.3 \%$ of the $X_{\max }$ is reached and $\mathrm{t}_{2}$ is the time [h] in which 63.2 $\%$ of the $X_{\max }$ is reached. According to the exposed, a first approximation of the parameter $T_{p}$ is to consider the time in which a change is produced from the initial conditions $\left(63.2 \%\right.$ of $\left.\left(X_{\max }-X_{0}\right)\right)$. As $X_{0}$ is generally much less than $X_{\max }$, it can be neglected. Then, $T_{p}$ can be estimated as the time in which $63.2 \%$ of $X_{\max }$ is reached.

We compared the FOPDT model with the L Model. Equations 9 and 10 show the L model in its differentiated and integrated form (Mitchel et al. 2006), respectively:

$$
\begin{gathered}
\frac{d X}{d t}=\mu_{\max }\left(1-\frac{X}{X_{\max }}\right) X \quad ; \quad X(0)=X_{0} \\
X(t)=\frac{X_{\max }}{1+\left(\frac{X_{\max }}{X_{0}}-1\right) \exp ^{-\mu_{\max . t}}} \quad(10)
\end{gathered}
$$

Where $\mu_{\max }$ : Maximum Specific growth rate $\left[\mathrm{h}^{-1}\right]$. This model presents three important parameters: $X_{0}, X_{\max }$ and $\mu_{\max }$, where $X_{0}$ is the inoculum used in the experiment therefore it is a known value, whereas $X_{\max }$ and $\mu_{\max }$ have to be calculated by regression. 
so LA generation speed has a direct relationship with the microbial growth rate. For the production of LA,

$$
\frac{d P}{d t}=Y_{p / x} \frac{d X}{d t}+m_{p} X \quad P(0)=0
$$

Where: dP/dt: Lactic Acid formation rate $\left[\mathrm{g} . \mathrm{l}^{-1} \cdot \mathrm{h}^{-1}\right] ; Y_{p / x}$ : Lactic Acid yield $\left[\mathrm{g} \mathrm{LA}\right.$. g biomass ${ }^{-1}$; and $m_{p}$ : Coefficient for LA production related to maintenance metabolism [g LA . g biomass ${ }^{-1}$. hour]. The parameters $Y_{p / x}$ and $m_{p}$ will be known by regression, since the microbial growth rate could be adjusted to the proposed models. Equation 11 is rearranged to express $\mathrm{P}$ as a function of time, $\mathrm{P}(\mathrm{t})$, applying integral:

$$
\int_{0}^{P} d P=Y_{p / x} \int_{X_{0}}^{X} d X+m_{p} \int_{0}^{t} X(t) d t
$$

The kinetics of metabolite generation can be classified into three categories according to the values of the $Y_{p / x}$ and $m_{p}$ coefficients. When $Y_{p / x} \neq 0$ and $m_{p}=0$, the metabolite has a direct relationship with growth, being Type 1 ; When $Y_{p / x} \neq 0$ and $m_{p} \neq 0$, the metabolite has a mixed relationship with microbial growth and concentration of microorganism, being Type 2; and when $Y_{p / x}=0$ and $m_{p} \neq 0$, the metabolite has no relationship to microbial growth, being Type 3 (Gaden 2000). In the present work we applied the Type 1, since LA production is stabilized at a constant value, without presenting an increasing production in a linear way indefinitely, a situation that would occur in the case of $m_{p} \neq 0$. So, the production of LA when $Y_{p / x} \neq 0$ and $m_{p}=0$ is expressed as:

$$
\int_{0}^{P} d P=Y_{p / x} \int_{X_{0}}^{X} d X
$$

In the case of the $\mathrm{L}$ model, replacing equation 10 into equation 13 and solving results:

$$
P(t)=Y_{p / x}\left[\frac{X_{\max }}{1+\left(\frac{X_{\max }}{X_{0}}-1\right) \exp ^{-\mu_{\max . t}}}-X_{0}\right]
$$

$$
P(0)=0
$$

The parameters $Y_{p / x}$ and $m_{p}$ can vary with fermentation conditions. This coefficients have been widely studied in fermentative processes with bacteria of the genus Lactobacillus (Altiok et al. 2006), but not using fungi to obtain LA.

\subsubsection{Data analysis.}




\section{Results and discussion}

\section{Application and description of the proposed model}

The procedure applied to arrive at our proposed modification of the LP equation is described below:

1. We took an example of Fungal Submerged Fermentation (FSF) of corn waste stream using $R$. oryzae to obtain LA from the work of Jin et al. (2005) and obtained the experimental points of biomass and LA generation.

2. We fitted the L model, widely used and known in microbial growth kinetics, and then the L model combined with the LP model (L-LP model) for LA production, as traditionally done. We obtained Figure 1 and the parameters shown in Table 1.

Figure 1 about here

\section{Table 1 about here}

Table 1 shows that the model applied in biomass growth fits adequately according to the $\mathrm{R}^{2}$ value of $91.37 \%$, when combining the $\mathrm{L}$ model with the LP model for the production of LA, there is a clear delay time that can be observed in Figure $1 \mathrm{~b}$ and also the greatly reduced value of $\mathrm{R}^{2}$, showed in Table 1 .

3. Based on the observations made in step 2, we decided to apply the FOPDT model on biomass and LA production, in order to know the time evolution characteristics of the experimental responses $T_{p}$ and $T_{0}$ (equations 7 and 8), so as to be able to study the observed lag between biomass and LA. The results are shown in Figure 2 and Table 2. The FOPDT equation for the LA production used was:

$$
P(t)= \begin{cases}P_{0} \cdot \exp ^{-\left(t-T_{0}\right) / T_{p}}+P_{\max }\left(1-\exp ^{-\left(t-T_{0}\right) / T_{p}}\right) & \text { for } t \geq T_{0} \\ P=P_{0} & \text { for } t<T_{0}\end{cases}
$$

Where $\mathrm{P}(\mathrm{t})$ is the LA concentration obtained for a specific amount of time $\left[\mathrm{g} \cdot \mathrm{l}^{-1}\right] ; P_{\max }$ : 
delay time for LA production and $T_{p}[\mathrm{~h}]$ is the time constant of the process which provides information on the speed of growth up to $P_{\max }$. In the case of LA production $P_{0}=0$, since at the time $\mathrm{t}=0$ of the inoculation of the microorganism, lactic acid has not yet been produced. It should be noted that $Y_{p / x}=P_{\max } /\left(X_{\max }-X_{0}\right)$, so that by applying the FOPDT model, the value of LA formation yield with respect to cell biomass could be obtained.

Figure 2 about here

By applying the FOPDT model to the data, the fitting improved significantly reflecting an $\mathrm{R}^{2}$ for biomass data that went from $91.37 \%$ to $98.37 \%$ and for LA production went from $38.72 \%$ 

phenomena associated with LA production and also to obtain an increase in the $\mathrm{R}^{2}$ value, which leads to a better representation of the reality of fermentations. The proposed modification is expressed by:

$$
\frac{d P}{d t}=Y_{p / x} \frac{d X}{d t}\left(t-T_{d}\right) \quad \text { for } T_{d} \geq 0
$$

250

Where $T_{d}[\mathrm{~h}]$ is the time difference between the time when LA production and the biomass generation starts. This modification is based on the better fit observed in the LP model when applying the $T_{d}$ between the biomass and LA adjustments. In addition, the LP model, by omitting this delay time, omits biological phenomena that directly affect the time to obtain LA. The proposed modification probably also functions when $m_{p} \neq 0$, but has not been tested in the present work, as LA production has a direct relationship with microbial growth.

The L Model has the parameter $\mu_{\max }$, but it must be kept constant in the microbial and LA production kinetics, otherwise it would not adequately represent the phenomenon under study. Therefore, by incorporating the parameter $T_{d}$ in the L-LP model (equation 14), the system can be represented more faithfully, achieving an increase in the $\mathrm{R}^{2}$, resulting in:

$$
P(t)= \begin{cases}Y_{p / x}\left[\frac{X_{\max }}{\left.1+\left(\frac{X_{\max }}{X_{0}}-1\right) \exp ^{-\mu_{\max } \cdot\left(t-T_{d}\right)}-X_{0}\right]}\right. & \text { for } t \geq T_{0} \\ P(0)=P_{0}=0 & \text { for } t<T_{0}\end{cases}
$$

On the other hand, the FOPDT model, which already incorporates the $T_{0}$ parameter, offers the possibility of easily modifying and studying the time in which neither biomass nor LA is produced, without the need to modify or add parameters. Replacing equation 6 into equation 16 and solving, results:

$P(t)=\left\{\begin{array}{lr}Y_{p / x}\left[\left(X_{0} \cdot \exp ^{\left.\left.-\left[t-\left(T_{0}+T_{d}\right)\right] / T_{p}+X_{\max }\left(1-\exp ^{-\left[t-\left(T_{0}+T_{d}\right)\right]} / T_{p}\right)\right)-X_{0}\right]}\right.\right. & \text { for } t \geq T_{0} \\ m_{p} X_{0} t & \text { for } t<T_{0}\end{array}\right.$ model incorporating the parameter $T_{d}$ (FOPDT-LP with delay).

As it is known, $T_{0}=f$ (microorganism physiology, environment and type of inoculum), so using the L-LP with delay model or FOPDT-LP with delay model could decrease the $T_{d}$, modifying the fermentation conditions, and thus optimize the fermentation. 

example of item 3.1 are shown below: 1) to $94.57 \%$ (see Table 3). This shows that by adding the parameter $T_{d}$ to the LP equation, the approximation of the model with the experimental points is significantly improved. In the case of the FOPDT-LP with delay model the fit is the same as if we use the FOPDT model alone, so from here on we will show the FOPDT fit without combining it with LP to make it easier to identify the models. the other works chosen. Table 4 shows the parameters obtained for biomass generation using the L model and the FOPDT model, and the parameters obtained for LA production, using on the one hand the traditional L-LP model, and on the other hand the FOPDT model, with which we obtain the value of the parameter $T_{d}$, which is used in the logistic model combined with LP (equation 17), a model that we call L-LP with delay. It can be observed that in all cases what we have been describing is fulfilled, which clearly affirms and validates our proposal.

Table 4 about here

\section{Analysis of models adjustments}

To make clear the advantages of our proposal, bar charts were generated showing comparative $\mathrm{R}^{2}$ results for each model in the case of biomass generation and LA production. Figure 4a clearly shows that in biomass generation, the FOPDT model in the first four examples improves the fit with respect to the logistic model, while in the last three examples it is observed that the logistic model has a better fit, although the difference is minimal, so we can say that the FOPDT model is very useful and simple for modeling biomass generation. 

model and Logistic combined with LP with delay model (L-LP with delay). In all cases, the L-LP model is the worst fit, even reaching an $\mathrm{R}^{2}$ of less than $40 \%$ in the example where corn was used as fermentation substrate. This shows that an improvement is needed in the model that has been traditionally used and was supposed to give good results. This is evidenced by the better adjustments shown in all cases, both with the FOPDT model and with the L-LP with delay, showing that the proposal made in this work achieves a substantial improvement in the mathematical adjustment with respect to the traditional L-LP model. the analyses carried out in each case.

\section{Analysis of $T_{d}$ parameter} each case:

Table 5 compares the fermentation conditions of the works studied with the $T_{d}$ values obtained in

In Table 5 can be seen that they are all fermentations in liquid medium, so this would not change the $T_{d}$ value. The big difference in the $T_{d}$ value that is observed when comparing the type of microorganism. It is seen that when using a fungus such as $R$. oryzae, the $T_{d}$ value is $8 \mathrm{~h}$, a much higher value than when using LAB, such as L. delbruecki $\left(T_{d}=1 \mathrm{~h}\right)$, L. casei $\left(T_{d}=3 \mathrm{~h}\right)$ and L. rhamnosus $\left(T_{d}=2 \mathrm{~h}\right)$. This may be due to the fact that fungal growth is in the form of mycelium, which generates an increase in the viscosity of the liquid medium, which hinders the growth of the fungus, and therefore could slow down the generation of LA (Xu et al. 2017). In contrast, bacterial growth in liquid media does not bring about large changes in viscosity, so LA generation and secretion would be faster. In addition, fungi in general are not nutritionally demanding, so nutrients should not limit LA production, although in the experiment each byproduct (which provides the carbon source) was reinforced with nitrogen sources (yeast extract), amino acids (peptone) and minerals $\left(\mathrm{K}^{+}\right.$and $\left.\mathrm{Mg}^{+2}\right)$, so in this case nutrients would not be a limiting factor.

\section{Study Contributions}

The present study demonstrates that the Luedeking and Piret model is deficient to represent adequately the LA obtention by biotechnological process. Then, by adding the parameter $T_{d}$, is possible to analyze the time delay between biomass and LA production. The kinetic constants for microbial growth 
323 were obtained. The fit of the FOPDT model is comparable and in some cases even superior to the Logistic model. This shows the versatility and usefulness of the FOPDT model, which is widely known and used by

325 process engineers in industrial production plants because of their mathematical simplicity. For the mathematical model of LA production, the $T_{d}$ parameter added to the Luedeking and Piret model achieved

327 a significant improvement in $\mathrm{R}^{2}$. Furthermore, the $T_{d}$ parameter allows the study of the delay phenomenon between the generation of biomass and the metabolite in question, which has not been clearly defined so

329 far. With this proposal, we show the advantages of using the FOPDT model in a complementary way to the

330 Logistic model, since it allows the study of a phenomenon that the Logistic model alone does not allow.

331 Although the $T_{d}$ was obtained by approximating the experimental biomass and LA data with the FOPDT model, this delay time could also be used in the Logistic combined with Luedeking and Piret model, which

333 is a very novel contribution and implies an advance in the mathematical modeling of LA production. It's important to mention that the kinetic growth and the LA production have the same $T_{p}$ value, while they differ in the $T_{0}$ value, both will follow a variation with the same time constant. This reveals the adequacy of the Luedeking and Piret with delay model that we propose in this paper. In future work we will apply the Luedeking and Piret with delay model to other bioprocesses. 
Funding No funding was received for conducting this study. included in this published article and it supplementary information file.

344 literature search. MCG, GS and SEN were involved in the analysis and interpretation of data. MCG and

345 SEN drafted the manuscript. The study was supervised by GS, OAO and SEN. All authors read and 346 approved the final manuscript. performed by any of the authors. the study. 
352 Altık, D., Tokatl, F., and Sebnem, H. (2006) Kinetic modelling of lactic acid production from whey by

353 Lactobacillus casei ( NRRL B-441 ).J. Chem. Technol. Biotechnol.81, 1190-1197.

354 Amrane, A. (2001) Batch cultures of supplemented whey permeate using Lactobacillus helveticus :

355 unstructured model for biomass formation, substrate consumption and lactic acid production.Enzyme

356 Microb. Technol.28, 827-834.

357 Arino, O., Hbid, M.L., and Dads, E.A. (2006) Some general results and remarks on delay differential

358 equations.In Delay Differential Equations and Applications NATO Science Series,(The Netherlands:

359 Springer Dordrecht),pp.31-40.

360 Balannec, B., Bouguettoucha, A., and Amrane, A. (2007) Unstructured model for batch cultures without

361 pH control of Lactobacillus helveticus - Inhibitory effect of the undissociated lactic acid.Biochem. Eng.

362 J.35, 289-294.

363 Berry, A.R., Franco, C.M.M., Zhang, W., and Middelberg, A.P.J. (1999) Growth and lactic acid

364 production in batch culture of Lactobacillus rhamnosus in a defined medium.Biotechnol. Lett.21, 163-

365167.

366 Bouguettoucha, A., Balannec, B., Nacef, S., and Amrane, A. (2007) A generalised unstructured model for 367 batch cultures of Lactobacillus helveticus.Enzyme Microb. Technol.41, 377-382.

368 Bugge, M.M., Hansen, T., and Klitkou, A. (2016) What Is the Bioeconomy? A Review of the

369 Literature.Sustainability8, 691.

370 Crater, J.S., and Lievense, J.C. (2018) Scale-up of Industrial Microbial Processes.FEMS Microbiol.

371 Lett.365, fny138.

372 Gaden, E.L. (2000) Fermentation process kinetics.Biotechnol. Bioener.67, 629-635.

373 Germec, M., and Turhan, I. (2020) Enhanced production of Aspergillus niger inulinase from sugar beet

374 molasses and its kinetic modeling.Biotechnol. Lett.42, 1939-1955.

375 Gonzalez, K., Tebbani, S., Lopes, F., Thorigné, A., Givry, S., Dumur, D., and Pareau, D. (2016)

376 Modeling the continuous lactic acid production process from wheat flour.Appl. Microbiol.

377 Biotechnol.100, 147-159.

378 Grand View Research (2019) Lactic Acid Market Size Worth \$8.77 Billion By 2025 | CAGR: 18.7\%.

379 Gündüz, M. (2005) Lactic acid production by Lactobacillus casei NRRL B-441 immobilized in chitosan 380 stabilized Ca-alginate beads.

381 Herryman Munilla, M., and Blanco Carracedo, G. (2005) Ácido láctico y poliláctico: Situación actual y

382 tendencias.ICIDCA Sobre Los Deriv. La Caña AzúcarXXXIX, 49-59.

383 Huang, L.P., Jin, B., Lant, P., and Zhou, J. (2005) Simultaneous saccharification and fermentation of 
potato starch wastewater to lactic acid by Rhizopus oryzae and Rhizopus arrhizus.Biochem. Eng. J.23, 265-276.

Inkinen, S., Hakkarainen, M., Albertsson, A.C., and Södergård, A. (2011) From lactic acid to poly(lactic acid) (PLA): Characterization and analysis of PLA and Its precursors.Biomacromolecules 12, 523-532.

Jin, B., Yin, P., Ma, Y., and Zhao, L. (2005) Production of lactic acid and fungal biomass by Rhizopus fungi from food processing waste streams.J. Ind. Microbiol. Biotechnol.32, 678-686. Luedeking, R., and Piret, E.L. (1959) A kinetic study of the lactic acid fermentation. Batch process at controlled pH.J. Biochem. Microbiol. Technol. Eng.1, 393-412.

Mitchel, D.., Luz, L.F.., Berovič, M., and Krieger, N. (2006) Approaches to Modeling SSF Bioreactors.In Solid-State Fermentation Bioreactors,D.A. Mitchell, M. Berovič, and N. Krieger, eds.(Springer, Berlin, Heidelberg),pp.159-178. J., et al. (2019) Sustainable biosynthesis of curdlan from orange waste by using Alcaligenes faecalis: A systematically modeled approach.Carbohydr. Polym.205, 626-635.

Palaniraj, R., and Nagarajan, P. (2012) Kinetic Studies In Production Of Lactic Acid From Waste Potato Starch Using Lactobacillus casei.Int. J. ChemTech Res.4, 1601-1614.

400 Rodrí́guez León, J.A., de Carvalho, J.C., Pandey, A., Soccol, C.R., and Rodríguez Fernández, D.E.

401 (2017) Kinetics of the Solid-State Fermentation Process.In Curr. Dev. Biotechnol. Bioeng.,pp.57-82.

402 Rosero-chasoy, G., Durán-páramo, E., and Chairez, I. (2020) Time-delay mathematical model of lagged 403 lactic acid production using agro-industrial wastes as substrate.Appl. Math. Model.83, 136-145.

404 Saat, M.N., Annuar, M.S.M., Alias, Z., Chuan, L.T., and Chisti, Y. (2014) Modeling of growth and 405 laccase production by Pycnoporus sanguineus.Bioprocess Biosyst. Eng.37, 765-775.

406 Sardella, M.F., Serrano, M.E., Camacho, O., and Scaglia, G.J.E. (2020) Improvement of linear algebra 407 controllers using sliding surface concepts : applications to chemical processes.IEEE Lat. Am. Trans.100, $408 \quad 1-8$.

409 Webb, C., and Manan, M.A. (2017) Design Aspects of Solid State Fermentation as Applied to Microbial 410 Bioprocessing.J. Appl. Biotechnol. Bioeng.4, 511-532.

411 Xu, Q., Fu, Y., Li, S., Jiang, L., Rongfeng, G., and Huang, H. (2017) Integrated transcriptomic and 412 metabolomic analysis of Rhizopus oryzae with different morphologies.Process Biochem. 
413 Table 1: Biomass and LA mathematical model parameters obtained from L model and LP model fitting.

\begin{tabular}{cccccccc}
\hline \multirow{2}{*}{ Medium } & Kinetic & Model & $X_{0}$ & $X_{\max }$ & $\mu_{\max }$ & $Y_{p / x}$ & $\mathrm{R}^{2}$ \\
& & & & & & & \\
\hline \multirow{2}{*}{ Corn } & BIOMASS & L & \multirow{2}{*}{0.0014} & 5.37 & 0.9912 & - & 91.37 \\
\cline { 2 - 7 } & LA & L-LP & & & & & \\
\hline
\end{tabular}

414 
Table 2: Biomass and LA mathematical model parameters obtained from FOPDT model fitting.

\begin{tabular}{|c|c|c|c|c|c|c|c|c|c|}
\hline Medium & Kinetic & Model & $X_{0}$ & $X_{\max }$ & $P_{\max }$ & $T_{p}$ & $T_{0}$ & $T_{d}$ & $\mathrm{R}^{2}$ \\
\hline \multirow{2}{*}{ Corn } & BIOMASS & \multirow{2}{*}{ FOPDT } & \multirow{2}{*}{0.0014} & 5.37 & - & \multirow{2}{*}{6.62} & 4 & \multirow{2}{*}{8} & 98.20 \\
\hline & LA & & & - & 14.5 & & 12 & & 97.72 \\
\hline
\end{tabular}

416 
Table 3: Biomass and LA mathematical model parameters obtained from FOPDT and L with LP with delay models.

\begin{tabular}{|c|c|c|c|c|c|c|c|c|c|c|}
\hline Medium & Kinetic & Model & $X_{0}$ & $X_{\max }$ & $\mu_{\max }$ & $\mathrm{Y}_{p / x}$ & $T_{p}$ & $T_{0}$ & $T_{d}$ & $\mathrm{R}^{2}$ \\
\hline \multirow{2}{*}{ Corn } & \multirow{2}{*}{ LA } & FOPDT-LP with delay & & 5.37 & 0.9912 & \multirow{2}{*}{2.7} & \multirow{2}{*}{6.62} & 12 & \multirow{2}{*}{8} & 97.72 \\
\hline & & L-LP with delay & & - & - & & & 8 & & 94.57 \\
\hline
\end{tabular}

419 
Table 4: Biomass and LA mathematical model parameters obtained from the fitting of the works.

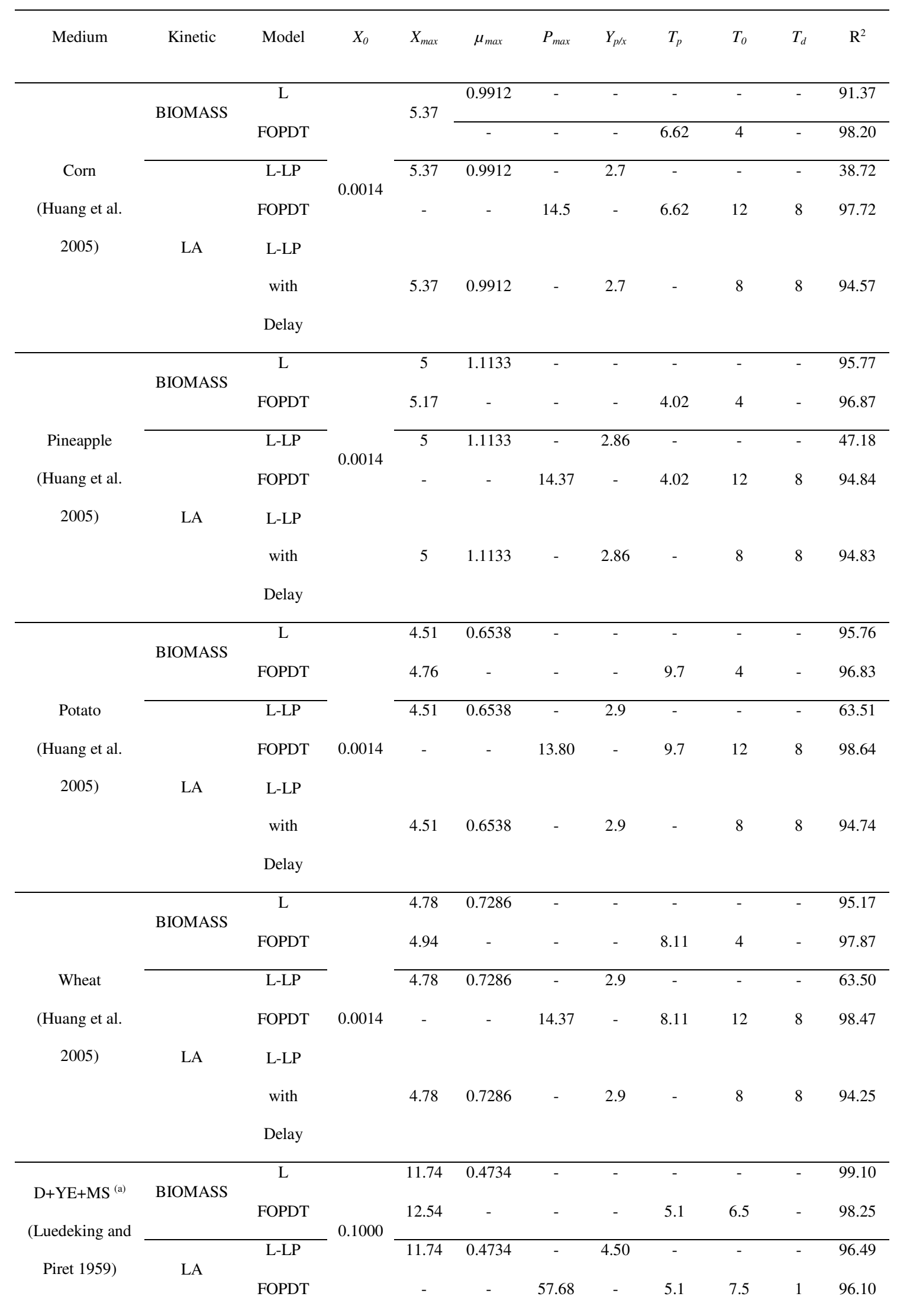




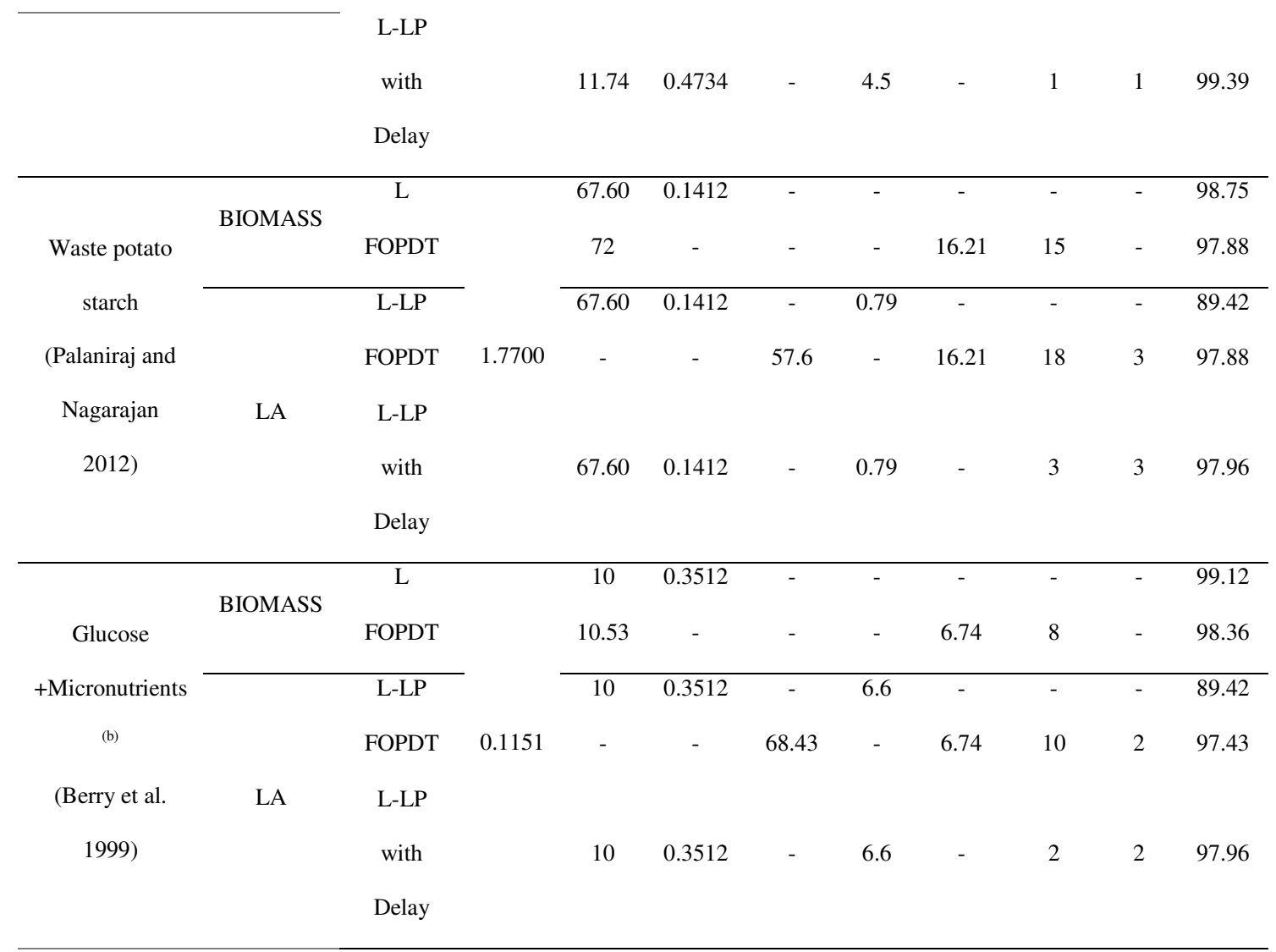

$421 \quad{ }^{(a)}$ Dextrose, yeast extract and mineral salts.

422 (b) Mineral salts, vitamins and amino acids. 
Table 5: Comparison of fermentation conditions of the works analyzed with the $T_{d}$ value.

\begin{tabular}{|c|c|c|c|c|c|c|c|}
\hline \multirow{3}{*}{ Author } & \multirow{2}{*}{\multicolumn{2}{|c|}{ Microorganism }} & \multirow{3}{*}{ Medium } & \multirow{3}{*}{ Nutrients } & \multirow{2}{*}{\multicolumn{2}{|c|}{ Environment }} & \multirow{3}{*}{$T_{d}$} \\
\hline & & & & & & & \\
\hline & Type & Inoculum & & & $\mathrm{T}^{\mathrm{o}}$ & $\mathrm{O}_{2}$ & \\
\hline
\end{tabular}

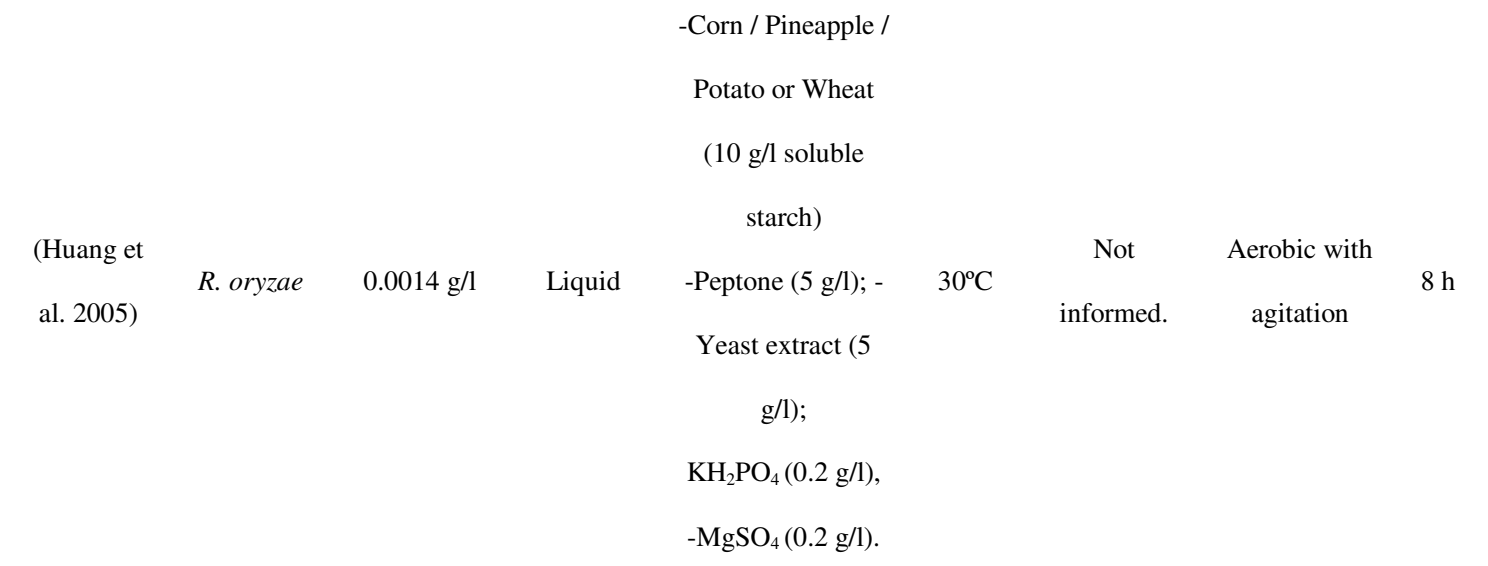

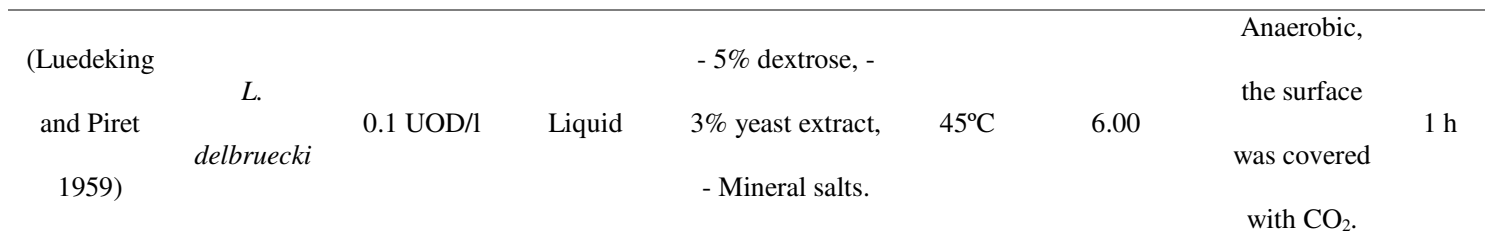

-Potato waste $(100$

(Palaniraj $\quad \mathrm{g} / \mathrm{l})$; - Yeast extract

\begin{tabular}{|c|c|c|c|c|c|c|c|}
\hline $\begin{array}{c}\text { and } \\
\text { Nagarajan }\end{array}$ & L. casei & $1.77 \mathrm{~g} / 1$ & Liquid & $\begin{array}{l}(12 \mathrm{~g} / \mathrm{l}) ; \text {-Enzyme } \\
\text { mixture }(10 \mathrm{ml} / \mathrm{l}) ;-\end{array}$ & $37^{\circ} \mathrm{C}$ & 6.00 & Anaerobic \\
\hline
\end{tabular}

2012)

Ammonium

chloride $(3 \mathrm{~g} / \mathrm{l})$.

-Glucose (80 g/l);

-Minerals salts

$\left(\mathrm{K}^{+}, \mathrm{Na}^{+}, \mathrm{Mg}^{+}\right.$,

\begin{tabular}{|c|c|c|c|c|c|c|c|}
\hline & \multirow{3}{*}{$\begin{array}{c}\text { L. } \\
\text { rhamnosus }\end{array}$} & \multicolumn{5}{|c|}{ Mn, $\mathrm{Fe}$} & \multirow{3}{*}{$\begin{array}{l}\text { Microaerobic } \\
\text { with } \\
\text { agitation }\end{array}$} \\
\hline & & $0.1151 \mathrm{~g} / 1$ & Liquid & $\mathrm{Co}^{++}, \mathrm{Cu}^{++}, \mathrm{Ni}^{++}$, & $40^{\circ} \mathrm{C}$ & 5.50 & \\
\hline & & \multicolumn{5}{|c|}{$\left.\mathrm{NH}_{4}^{+}, \mathrm{Zn}^{++}\right)$} & \\
\hline $\begin{array}{l}\text { (Berry et } \\
\text { al. 1999) }\end{array}$ & \multicolumn{7}{|c|}{-10 Vitamins } \\
\hline & \multicolumn{7}{|c|}{ types; -20} \\
\hline
\end{tabular}

Aminoacids types. 
426 Model (script line) and b). Experimental points of LA production ( ) with L Model combined with LP 427 model (script line).

Fig. 2: FSF Mathematical modeling: a). Experimental points of $R$. oryzae Biomass FOPDT Model (solid line) and b). Experimental points of LA production $(\checkmark)$ with FOPDT Model (solid line).

Fig. 3: FSF Mathematical modeling: Experimental points of LA production Lactic Acid ( $)$; FOPDT-LP with delay Model (dotted line) and L-LP Model with delay model (script and dotted line).

Fig. 4: Comparative results of the $\mathrm{R}^{2}$ for each model applied to: a). Cell biomass generation: Logistic model $(\square)$ and FOPDT model $(\square)$; and b). LA production: Logistic combined with LP model

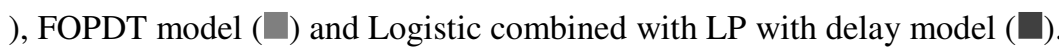



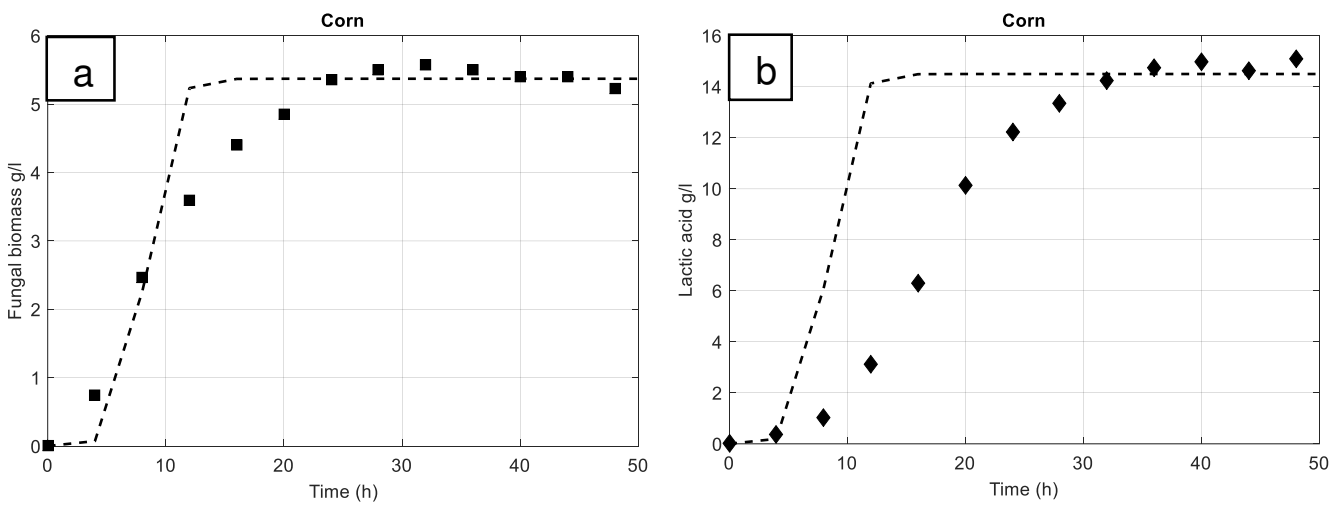

Fig. 1 

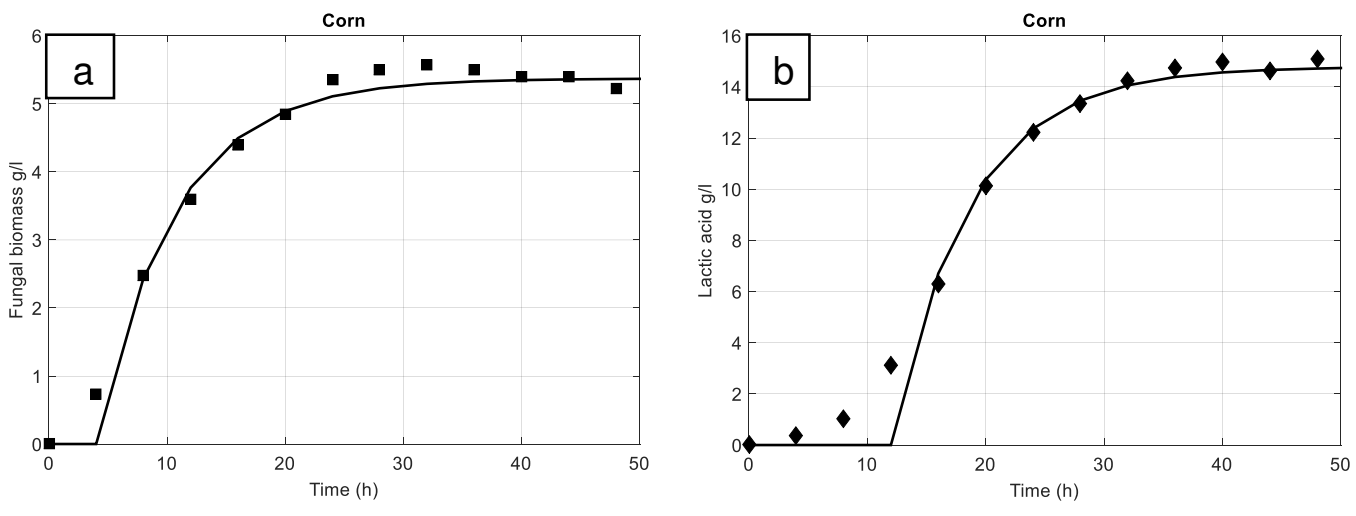

Fig. 2 


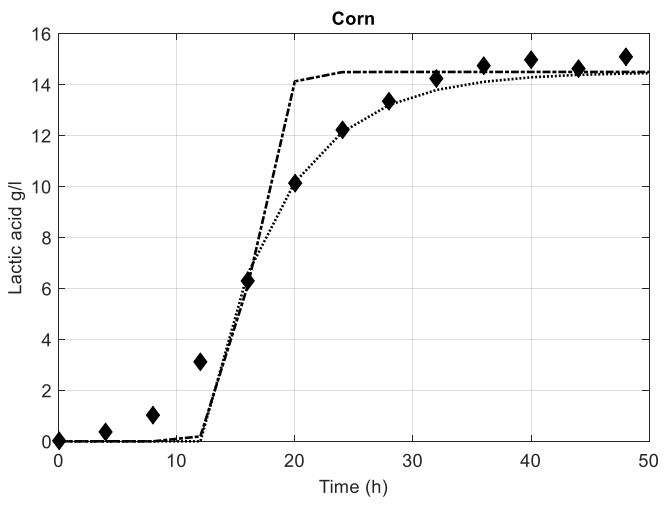

441

Fig. 3 


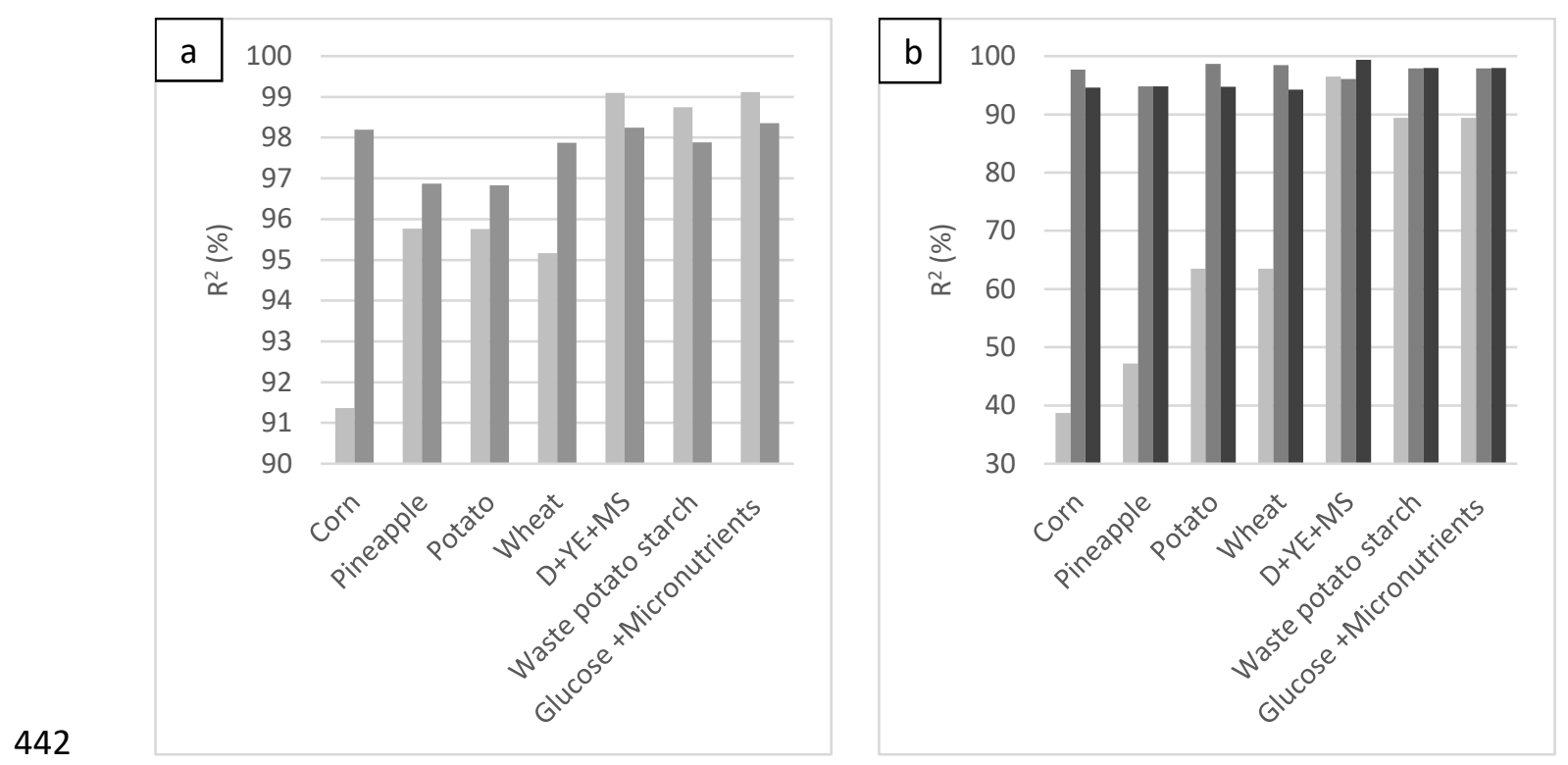

Fig. 4 


\section{Supplementary Files}

This is a list of supplementary files associated with this preprint. Click to download.

- BLSupplementaryInformationGroffetal.pdf 